\title{
Features of biogas technology application in severe climatic conditions
}

\author{
E. Arbuzova, S. Shcheklein \& V. Velkin \\ Department of Nuclear Power Plants and Renewables, \\ Ural Federal University, Russia
}

\begin{abstract}
The organic waste of the agriculture and food industry can be a potential resource for bioenergy and the potential of biomasses from different origins in the Russian Federation is significant. Biomass annual growth is comparable with traditional organic energy consumption, so the introduction of modern biotechnology is important.

The performed study of the effect of various factors on the biological treatment processes for a certain type of biomass (cattle manure) shows that it is possible to reduce processing time and to increase the biomass conversion degree at moderate energy consumption.

A biogas plant (BGP-100) was developed with a high level of thermal protection and heat recovery to make the biological treatment process of organic waste effective, even in areas with severe climate conditions.

A further development of biogas technology effectiveness may be the use of more active bacterial cultures and cogeneration plants combined with some types of thermal energy produced to recover technology needs.
\end{abstract}

Keywords: biogas, biomass, severe climatic conditions.

\section{Introduction}

Mankind has been widely using the fuels of vegetable origin from the very beginning of civilization. Nowadays, a large amount of production and waste of agriculture, including animal husbandry and the food industry, is added to natural biomass (grass, wood, etc.). Current technologies of acid and fermentative hydrolysis, gas production, and pyrolysis allow to produce motor fuels, biogas, 
and high-energy solid fuels from biomass, as opposed to conventional burning which can provide just heat energy.

Natural biomass is the wealth of our planet, but plenty of organic waste of agriculture and food industry can be not only the potential resource of bioenergy but an environmental hazard. Conventional ways of organic waste processing are complicated enough in terms of technology and demand high power inputs. It gives one more incentive to introduce the technologies of fuel production from biomass.

The production of methane in biogas reactors can not only solve the problem of waste utilization but supply agricultural enterprises with self-made cheap energy resource. Biogas can be used as a fuel for household gas devices, gas water heaters, air heaters. It also can be used for electric power generation in gas-fired plants.

The anaerobic methane generation from biomass is provided by the enzymatic ferment microorganisms which are present in the animal dung. The same process of organic decomposition we can meet in natural conditions of swamp, soil or animal bowels. Without oxygen or other energetically preferable electron acceptor access the bacteria have to use organic carbon and produce the most restored hydrocarbon compound - methane. Bacteria use the same carbon as an electron donor oxidizing it up to carbon-dioxide gas.

During the sequential process of organic decomposition various groups of bacteria destroy the carbon-carbonic bonds in the organic molecules. The process of anaerobic decomposition of nearly each organic substance can be divided into four stages:

1. Hydrolysis of complex biopolymer molecules (proteins, lipids, polysaccharides etc.) into simpler monomers and oligomers such as amino acids, carbohydrates, fatty acids etc.

2. Fermentation of the produced monomers to simpler acids and alcohols. Hydrogen and carbon acid are also produced at this stage.

3. Acetogenic stage of methane precursors (acetate, hydrogen, carbon acid) production.

4. Methanogenic stage leading to the complex organic substance decomposition end product - methane.

It should be mentioned that the stages of hydrolysis and fermentation are not legibly separated because the hydrolyzing microorganisms use the hydrolysis products for their growth.

Several hundreds of microorganism species participate in the decomposition process. Various groups of microorganisms are linked into complex food chains, for example methanogenic bacteria can't grow without the trophic link with the bacteria of previous stages. The methanogenic bacteria in turn determine the anaerobic microorganisms growth rate.

The methanogenic fermentation of concentrated organic waste such as animal dung, sewage sediment and domestic waste can begin in anaerobic conditions without microorganism additive because the needed microflora is always available from the waste and environment. But when starting an anaerobic bioreactor, some active ferment is added to the organic waste to intensify the process. 
The methanogenic fermentation can run within the range from $0^{\circ}$ to $97^{\circ} \mathrm{C}$, but the optimum temperature is $35-40{ }^{\circ} \mathrm{C}$ for the methophilic process and $50-55^{\circ} \mathrm{C}$ for the thermophilic process.

\section{Methods of increasing the biogas plant efficiency}

The most important problem of the biotechnology feasibility is the biogas plant efficiency dependence on climatic factors. First of all: its dependence on the ambient temperature, as in modern biogas plants bacterial methanogenesis occurs under special conditions (oxygen absence, high temperature, high humidity, the need for continuous homogenization, etc.).

According to Baader et al. [1] the energy from biogas does not cover the cost to maintain the biogas plant working conditions. Therefore, biotechnology organic waste purification are widely used only in regions and countries with a warm climate.

The our work purpose is the technology biogas plants energy efficiency analyze depending on climatic factors; the potential effectiveness determine of their use in the Russian Federation different regions; possible areas identify of optimization technologies biogas plants [2, 3].

Bailey and Ollis [5] and Biryukov [6] define the measure of energy efficiency of biogas technology, as the ratio of biogas $\left(Q_{g}\right)$ to the overall cost of energy required to produce it $\left(E_{o n}\right)$ :

$$
K_{\text {eff }}=\frac{Q_{g}}{E_{\text {on }}} .
$$

Accepted that technology is energy efficient, if $K_{\text {eff }}>1$, that is, received energy amount exceeds the total cost of software technology.

The biogas thermal energy:

$$
Q_{g}=b_{g}^{u} \cdot V_{g}, \text { МДж, }
$$

where $b_{g}^{u}$ - biogas calorific value, $\mathrm{MJ} / \mathrm{m}^{3}, V_{g}$ - produced biogas volume, $\mathrm{m}^{3}$.

In a general view the biogas yield produced from certain type raw can be determined as:

$$
V_{g}=\left(1-\varphi_{s}\right) \cdot M_{s} \cdot v_{u}^{\max } \cdot \alpha_{d e c}(\tau),
$$

where $v_{u}^{\max }$ - maximum specific biogas output, $\alpha_{d e c}(\tau)$ - decomposition coefficient (time function).

Table 1 shows the characteristic quantities and yield rate of biogas according Pantskhava and Pogarnov [7], Gaab [8] and Kovalev [9].

It is known that from cattle manure in the process inhibitors absence, optimum $\mathrm{pH} 6.5-7$, and raw material moisture 90-98\%, the yield of biogas reaches $45-55 \%$ of the total biogas yield for the cycle time. Optimal parameters processing cycles for the different modes of fermentation are shown in Table 2 according to [7-9]. 
Table 1: The characteristic quantities and output rates of biogas.

\begin{tabular}{|c|c|c|c|c|c|c|c|}
\hline \multirow{2}{*}{ Raw type } & \multirow{2}{*}{$\begin{array}{l}\text { The full biogas } \\
\text { yield } \mathrm{m}^{3} / \mathrm{t}\end{array}$} & \multirow{2}{*}{ Regime* } & \multicolumn{5}{|c|}{$\begin{array}{l}\text { Yield biogas percentage to the fermentation } \\
\text { duration, days }\end{array}$} \\
\hline & & & 10 & 15 & 20 & 40 & 60 \\
\hline $\begin{array}{l}\text { Cattle } \\
\text { manure }\end{array}$ & 300 & \begin{tabular}{|l|}
$\mathrm{P}$ \\
$\mathrm{M}$ \\
$\mathrm{T}$ \\
\end{tabular} & 24 & 36 & 48 & 82 & 95 \\
\hline Straw & 350 & \begin{tabular}{|l|}
$\mathrm{P}$ \\
$\mathrm{M}$ \\
$\mathrm{T}$
\end{tabular} & 29 & 38 & 45 & 88 & 96 \\
\hline Grass & 490 & \begin{tabular}{|l|}
$\mathrm{P}$ \\
$\mathrm{M}$ \\
$\mathrm{T}$
\end{tabular} & 87 & 99 & 100 & & \\
\hline
\end{tabular}

* P - psychrophilic, M - mesophilic, T - thermophilic regime.

Table 2: Optimal parameters processing cycles for the different modes of fermentation.

\begin{tabular}{|l|c|c|c|}
\hline Regime & $\begin{array}{c}\text { Optimum } \\
\text { temperature, } t_{j},{ }^{\circ} \mathrm{C}\end{array}$ & $\begin{array}{c}\text { Optimum } \\
\text { humidity, } \varphi_{s} .\end{array}$ & $\begin{array}{c}\text { Optimum } \\
\text { cycle, } \tau_{j} \text {, day }\end{array}$ \\
\hline Psychrophilic & 20 & $0.90-0.96$ & 75 \\
\hline Mesophilic & 35 & $0.90-0.96$ & 25 \\
\hline Thermophilic & 55 & $0.90-0.96$ & 15 \\
\hline
\end{tabular}

The technological scheme of the simplest biogas plant is shown in Figure 1. Thermal resistance $\mathrm{R}$ is assumed equal to 0.2 , then there is insulation missing.

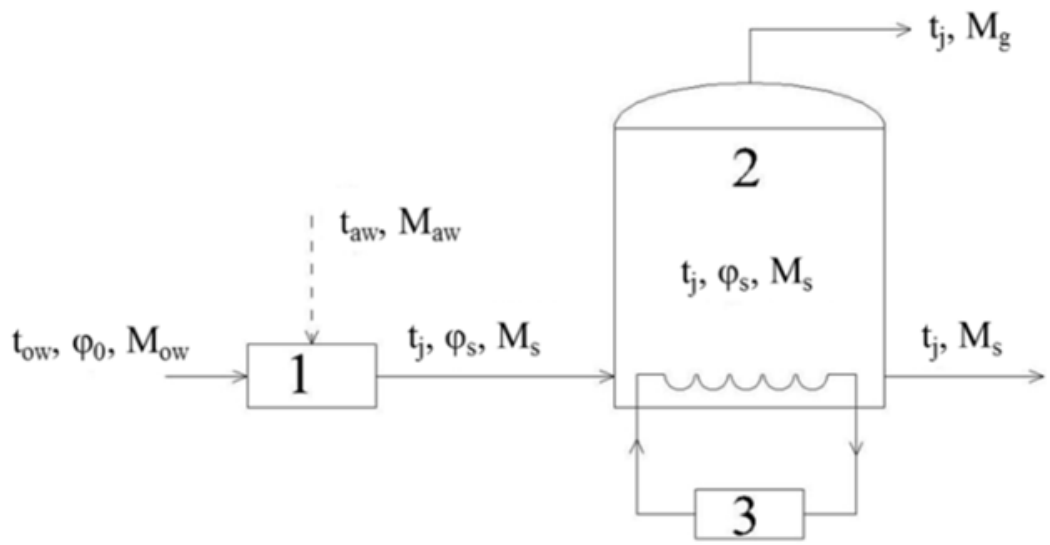

Figure 1: Technological scheme of the simplest biogas plant: 1 - mixer, 2 - bioreactor, 3 - heater. 
Organic waste with temperature $t_{o w}$, humidity $\varphi_{0}$ and mass rate $M_{o w}$ is mixed with additional water (temperature $t_{a w}$, mass rate $M_{a w}$ ) in the mixer 1 . Here the mixture (substrate) achieves the regime temperature $t_{j}$ which is $36^{\circ} \mathrm{C}$ for mesophilic, $55^{\circ} \mathrm{C}$ for thermophilic and $20^{\circ} \mathrm{C}$ for psychrophilic regime. Than the substrate with humidity $\varphi_{s}$ and mass rate $M_{s}$ enters the bioreactor 2 where it is decomposed into biogas with mass rate $M_{g}$ and residue. To support the regime temperature the heater 3 is used.

Energy consumption for own needs BGP technology $-E_{\text {on }}$ consists of heat flow $\left(Q_{o n}\right)$ for heating to the optimum temperature of biomass and water which diluting the biomass to the optimum humidity, compensation of heat losses from the bioreactor and other BGP equipment to the environment; consumption of electrical energy $\left(E_{o n}\right)$ for preparation (grinding, mixing), substrate transport, substrate delete, and periodic mixing of raw materials and the substrate in the BGP, work of the control system.

For an accurate assessment, energy consumption of all types (chemical, thermal, mechanical, electrical) should be reduced to one (for example thermal) equivalent:

$$
E_{o n}=E_{o n}^{h}+E_{o n}^{e l}
$$

Heat consumption for own needs of technology:

$$
E_{\text {on }}^{h}=Q_{\text {on }}=Q_{\text {heat }}+Q_{\text {loss }}, \mathrm{J},
$$

where $Q_{\text {heat }}=C_{s} \cdot M_{s} \cdot\left(t_{i}-t_{s}\right)$, J,

$Q_{\text {loss }}=\frac{F}{R} \cdot\left(t_{j}-t_{\text {env }}\right), \mathrm{J}$,

$t_{j}, t_{s}, t_{e n v}=$ substrate temperature in the reactor, substrate temperature at the bioreactor inlet, and environment temperature respectively, $C_{S}, M_{S}=$ substrate heat capacity and substrate mass in the bioreactor, $F, R=$ surface heat exchange with the environment and the temperature coefficient of resistance of the circuit thermal protection, respectively.

Electricity consumption for own needs technology:

$$
C_{\text {on }}=C_{\text {dis }}+C_{\text {pump } 1}+C_{\text {pump } 2}+C_{\text {mix }}+C_{\text {control }}, \mid
$$

where $C_{d i s}=a \cdot M_{s} \cdot \eta_{d i s}$ - energy consumption for mixing and grinding the substrate,

$C_{\text {pump } 1}=g \cdot M_{s} \cdot H_{s} \cdot \eta_{\text {pump } 1}$ - energy consumption for filling the bioreactor,

$C_{\text {pump } 2}=g \cdot M_{s} \cdot H_{s} \cdot \eta_{\text {pump } 2}$ - energy consumption for emptying bioreactor,

$C_{\text {mix }}=n_{j} \cdot g \cdot M_{s} \cdot H_{s} \cdot \eta_{m i x}$ - energy consumption for mixing in the bioreactor,

$n_{j}$ - the number of cycles during the mixing of the bioreactor, 
$H_{s}$ - height displacement of biomass for filling, emptying and mixing in the volume of the bioreactor, $\mathrm{m}$, $\eta_{\text {dis }}, \eta_{\text {pump } 1}, \eta_{\text {pump } 2}, \eta_{\text {mix }}-$ efficiencies of less than 0.8 .

Assessing the need for thermal energy to produce the required amount of electricity shall be subject to the modern electric power technology efficiency characterized by the efficiency of thermal power plants and nuclear power plants. Achieved level $-\eta_{e s}=0.35$ to determine the thermal equivalent of electrical energy consumed:

$$
E_{o n}^{e l}=\frac{C_{o n}}{\eta_{e s}}, \mathrm{~J}
$$

Since biogas technologies are designed to work within a year, it is advisable to determine their effectiveness in the annual cycle of work. In this case, the annual biogas yield and energy consumption for own needs of technology can be determined by integration of the corresponding functions, taking into account the temperature dependence of media and the outside air from the corresponding period [10].

In a general view:

$$
\begin{aligned}
& Q_{g}^{\text {year }}=b_{g}^{u} \int_{0}^{T_{\text {year }}}\left(1-\varphi_{s}\right) \cdot M_{s} \cdot v_{u}^{\max } \cdot \alpha_{d e c}(\tau) d \tau=b_{g}^{u} \cdot \overline{v_{u}} \cdot M_{s} \cdot\left(1-\varphi_{s}\right) \\
& E_{c H}=\int_{0}^{T_{\text {year }}} C_{s} \cdot M_{s} \cdot n_{j} \cdot\left[\frac{1-\varphi_{S}}{1-\varphi_{0}} \cdot\left(t_{j}-t_{s}\right)+\frac{\varphi_{s}-\varphi_{0}}{1-\varphi_{0}} \cdot\left(t_{j}-t_{w}^{a}\right)\right]+ \\
& \frac{F \cdot T_{\text {year }}}{R}\left(t_{j}-t_{\text {env }}(\tau)\right)+\eta_{\text {es }}^{-1} \cdot M_{s} \cdot g \cdot h \cdot\left[2 \cdot \eta_{\text {env }}^{-1} \cdot n_{j}+n_{\text {mix }} \cdot \eta_{\text {mix }}^{-1}\right] d \tau=C_{s} \cdot \\
& M_{s} \cdot n_{j} \cdot\left[\frac{1-\varphi_{s}}{1-\varphi_{0}} \cdot\left(t_{j}-t_{s}\right)+\frac{\varphi_{S}-\varphi_{0}}{1-\varphi_{0}} \cdot\left(t_{j}-t_{B}^{\not}\right)\right]+\frac{F \cdot T_{\Gamma \text { O Д }}}{R}\left(t_{j}-\overline{t_{H}}\right)+\eta_{e s}^{-1} \cdot M_{s} \cdot \\
& g \cdot h \cdot\left[2 \cdot \eta_{\text {pump }}^{-1} \cdot n_{j}+n_{\text {mix }} \cdot \eta_{\text {mix }}^{-1}\right]
\end{aligned}
$$

where $n_{j}=\frac{T_{y e a r}}{\tau_{j}}$, the annual number of downloads,

Thus:

$$
\overline{t_{\text {env }}}=\frac{1}{T_{\text {yaer }}} \int_{0}^{T_{\text {year }}} t(\tau) d \tau \text { - average ambient temperature. }
$$

$$
K_{\text {eff }}^{\text {year }}=\frac{n_{j}\left(1-\varphi_{s}\right) M_{s} \overline{v_{u}} b_{g}^{u}}{C_{s} M_{S} n_{j}\left[\frac{1-\varphi_{S}}{1-\varphi_{0}}\left(t_{j}-t_{s}\right)+\frac{\varphi_{S}-\varphi_{0}}{1-\varphi_{0}}\left(t_{j}-t_{w}^{a}\right)\right]+\frac{F T_{y e a r}}{R}\left(t_{j}-\bar{t}_{\text {env }}\right)+\eta_{e s}^{-1} M_{s} g h\left[\left(2 \eta_{p u m p}^{-1}\right) n_{j}+n_{m i x} \eta_{m i x}^{-1}\right]}
$$

It should be noted that for biogas digesters efficiency essentially depends on the scale factor, which determines the ratio of the volume and the heat transfer surface.

The results of calculation of the value of the scale factor for the reactor of cylindrical shape shown in Figure 2. 


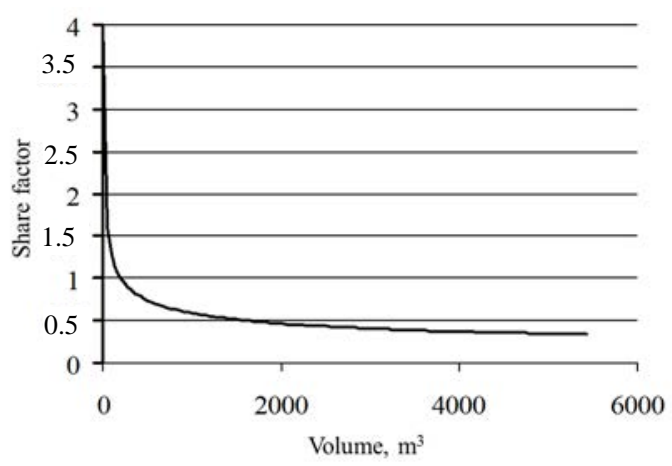

Figure 2: Scale factor for a reactor of cylindrical shape.

The reactor heat loss optimization according to the method [10], based on the solution of the conditions finding variation problem $d Q_{\text {pot }} / d V=\min$, provides a geometrical ratio, as follows:

$h_{\text {optimum }}=0,86 \cdot\left(\frac{M_{S}}{\rho}\right)^{1 / 3}$ - height of the reactor, $\mathrm{m}$,

$F_{\text {optimum }}=5,6 \cdot\left(\frac{M_{S}}{\rho}\right)^{2 / 3}$ - surface heat exchange with the environment, $\mathrm{m}^{2}$,

$d_{\text {optimum }}=1,22 \cdot\left(\frac{M_{s}}{\rho}\right)^{1 / 3}-$ diameter of the reactor, $\mathrm{m}$.

The influence of climatic factors on the bioreactor heat loss is caused due to the annual changes in ambient temperature.

The simplest type bioreactor efficiency is extremely low. We can link the bioreactor efficiency with ambient temperature through a unit which is used to indicate the severe climate - heating degree-days (HDD). This parameter is equal to the medium difference between the indoor and outdoor temperature throughout the heating season multiplied the duration of heating season.

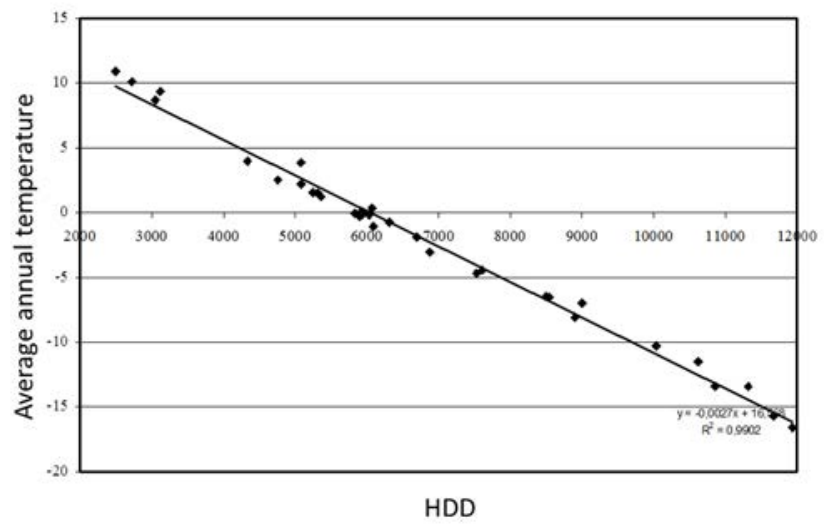

Figure 3: The dependence of the mean annual temperature of HDD. 
This dependence with an error of less than $2 \%$ is approximated by a linear equation: $t_{\text {env }}=16.5-2.7 \cdot 10^{3} \cdot$ HDD.

Figure 4 shows the results of calculations of the Keff from HDD for characteristic modes methanogenesis for flow sheet (Figure 1). The calculation is performed for the cattle manure by incipient wetness $\left(\varphi_{0}\right)$ of $70 \%$. The moisture content in the bioreactor $\left(\varphi_{S}\right)$ adopted by $91.4 \%$. Thermal insulation is absent $\mathrm{R}=0.2$.

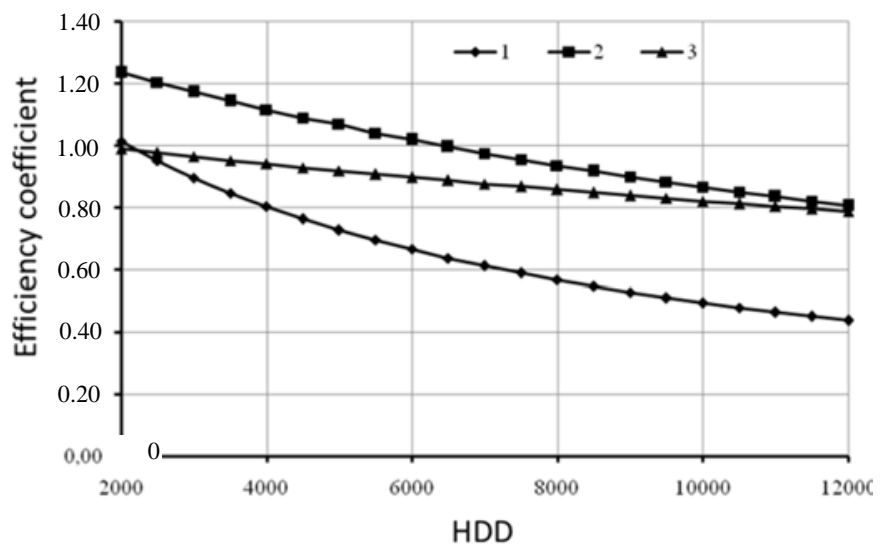

Figure 4: The dependence of the efficiency coefficient from the HDD for the simplest type bioreactor: 1 - psychrophilic methanogenesis regime; 2 - mesophilic methanogenesis regime; 3 - thermophilic methanogenesis regime.

The influence of climatic factors on the bioreactor heat loss is caused due to the annual changes in ambient temperature.

When heating degree-day (HDD) is more than 2000, efficiency coefficient becomes less than 1 for a number of regimes. This fact indicates that these regimes are inefficient. In the range of HDD more than 6000 some efficiency can be achieved only for mesophilic methane generation regime.

The analysis of certain factors that affect the organic waste biological treatment shows that the highest losses are connected with the heating of biomass and water which is added to substrate to achieve demanded humidity. Another source of losses is the maintenance of the desired temperature during the whole fermentation cycle period.

At the same time, to obtain the most favorable conditions for methanogenesis, as mentioned earlier, the starting biomass from the livestock farms must be converted to the liquid state by increasing its water content to the optimum level which is usually above $90 \%$.

Process flow diagram of a biogas plant using the effective thermal insulation ( $\mathrm{R}$ is more than 2) is shown in Figure 5. 


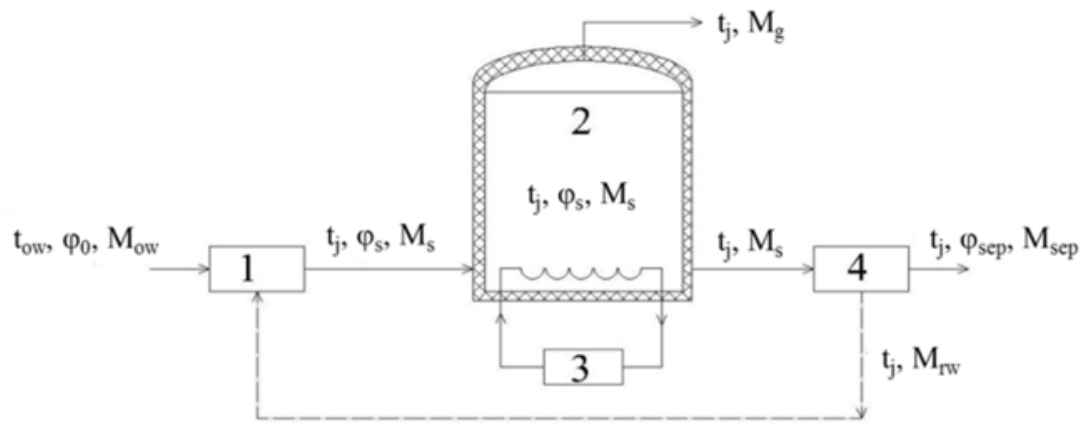

Figure 5: Process flow diagram of biogas technology with the recuperation of thermal energy by the method of separation and return of water to the cycle: 1 - mixer; 2 - bioreactor; 3 - heater; 4 - separator.

After the bioreactor 3 substrate enters the separator 4, where residue water is separated and with temperature $t_{j}$ and mass rate $M_{r w}$ comes into the mixer 1 .

Figure 6 shows the dependence of the biogas technology efficiency coefficient from the HDD for characteristic methanogenesis regimes for technological scheme shown in Figure 2. The temperature coefficient $(R)$ of circuit heat insulation thermal resistance in this case is assumed equal to 2 .

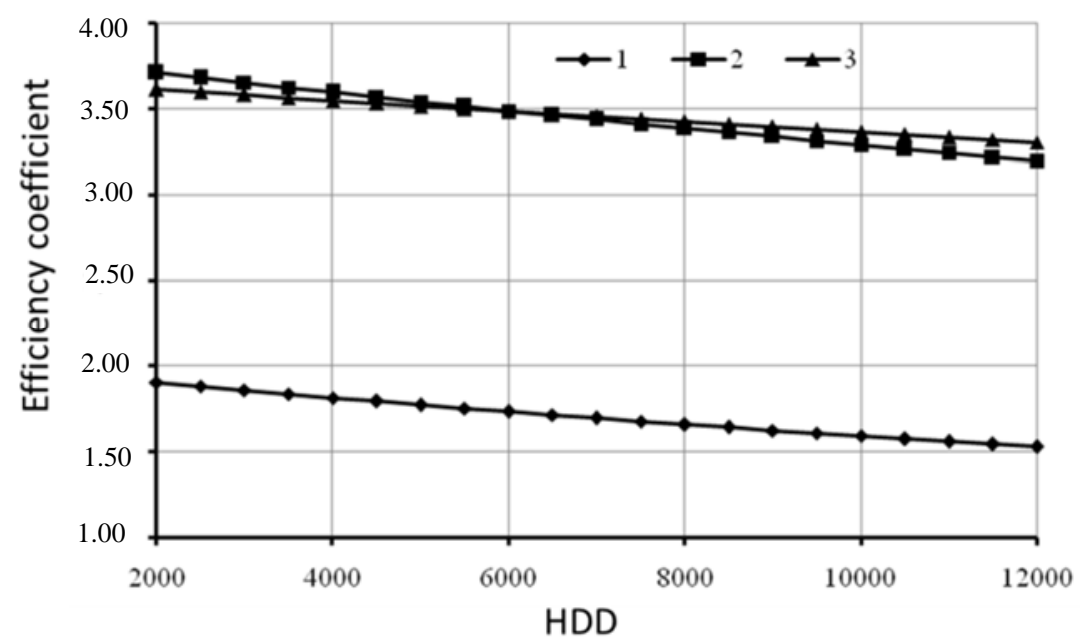

Figure 6: The dependence of the efficiency coefficient from the HDD for optimized bioreactor: 1 - psychrophilic methanogenesis regime; 2 - mesophilic methanogenesis regime; 3 - thermophilic methanogenesis regime. 
Figure 6 shows that the efficiency increases significantly and for modes 2 and 3 becomes comparable with the efficiency of production and delivery of traditional fossil fuels. The dependence of the biogas technology efficiency from the climatic factor for all methanogenesis regimes becomes mild. For the most fast (thermophilic and mesophilic) methanogenesis regimes the dependence of the biogas technology efficiency from the climatic factor does not exceed $10 \%$.

This process facilitates bacteria access to nutrients. As a result, the substrate decomposition rate increases. The disintegration methods can be:

- thermal - brief heating to the temperature below $100^{\circ} \mathrm{C}$ at normal pressure or above $100^{\circ} \mathrm{C}$ at increased pressure;

- chemical - addition of enzymes and catalysts, which can positively influence the process speed;

- biochemical; and

- physical - all mechanical methods including sonication.

The purpose of our work was the determination of sonication optimum for the biomass with $92 \%$ humidity.

Figure 7 shows the dependency of biogas components yield from the integral sonication dose. The data analysis shows that the maximum increase of biogas flammable components is $34-43 \%$ at the $5-10$ wh sonication dose.

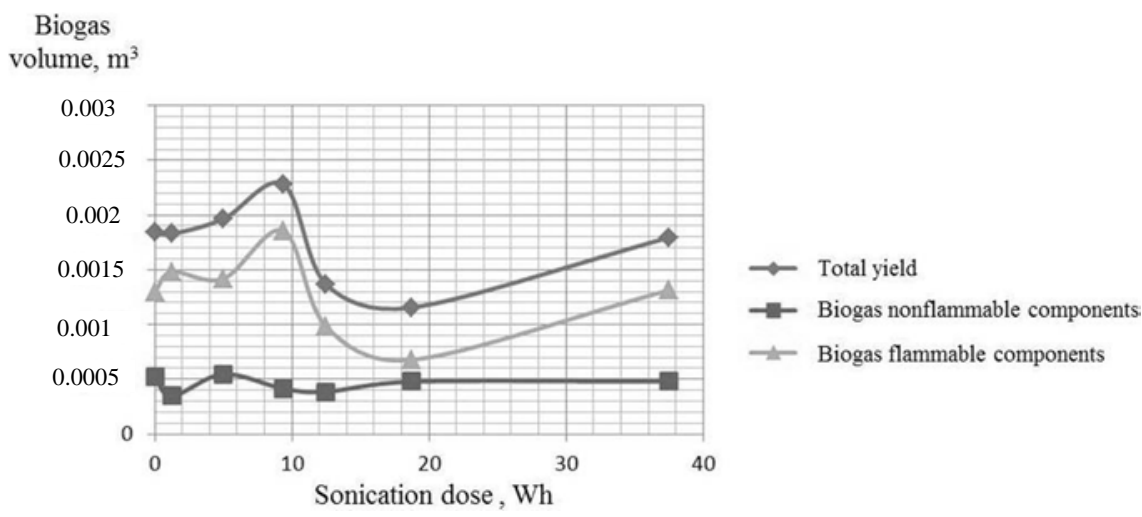

Figure 7: The biogas yield depending on the integral sonication dose.

Our investigations show that the sonication result in the biogas flammable components increase of 34-43\%. Sonication maximum efficiency is achieved at a moderate sonication dose. Additional energy costs for an ultrasound field with a maximum exposure time (half an hour) for the process total duration more than 20 days is less than $1 \%$. 


\section{Numerical model of the biogas reactor}

The reaction rate main influential parameters are the heating rate and the uniformity of temperature distribution. A computer model of the bioreactor preparatory tank was designed in terms of current investigation to estimate these parameters. The simulation was made with the help of SolidWorks and SolidWorks Flow Simulation software by Dassault Systems.

The main objective of the investigation was to create a computer model that can be used for the estimation of structural variations of a biogas reactor. The simulation results were compared with the performance of the bioreactor described in section 2 of this work.

The calculation showed that $13 \mathrm{~kW}$ of the heating system total power gives about 30 hours of heating time. Thus, numerical simulation shows the analogous heating time with the estimation formula. This fact can indirectly indicate the model validity. Almost the same time is needed to heat the biogas facility.

The next step was to examine different configurations of heating system. The number of heating elements varied from 10 to 33 for the same power input. The heating system of 33 elements showed the best results. This configuration of heating system allows to achieve needed temperature during the relatively small time period. The thermal gradient in the tank volume did not exceed $0.5^{\circ} \mathrm{C}$, so the heating uniformity was also achieved.

Through research the department of Atomic power plants and renewables, Ural Federal University, together with "Avanguard" PLC and “Gildia M" Ltd. have designed a biogas plant (the BGP-100) to produce methane from animal waste. The facility is intended to operate in the conditions of moderate and frigid climatic zones of Russia characterized by low temperatures. The facility flow diagram is shown in Figure 8.

The organic waste is accumulated in the dung collector. The supply pump sends it in the grinder system (1), than grinded dung comes into the suction tank (2) of the anaerobic reactor. After the stage of preparation it is transported into the main tank of the anaerobic reactor (3) by the substrate pump. The anaerobic reactor has a mixing system with electric drive (4) which improves the uniformity of the gasification process and heating system (12) which maintains assigned temperature regime. Biogas is removed from the reactor with gas compressor (5) to the dehumidification system (6), and the used substrate comes into the separation system with the help of the pump. Dry biogas is accumulated in the gasholder (7), from where it can be sent to the consumer (8). Decontaminated organic waste from the anaerobic reactor (3) comes to the separation system (9). Separated water is sent back in the bioreactor (3) with the regeneration pump (10). The off-balance water is removed from the cycle through the purification and sterilization system, and some of waste heat is reused for the tank heating. Produced biofertilizers additionally processed (11) for high temperature or ultrasonic.

The BGP-100 is made on the basis of composite materials. This is the reason for its longevity and high efficiency ratio when the BGP-100 used under severe climatic conditions. 


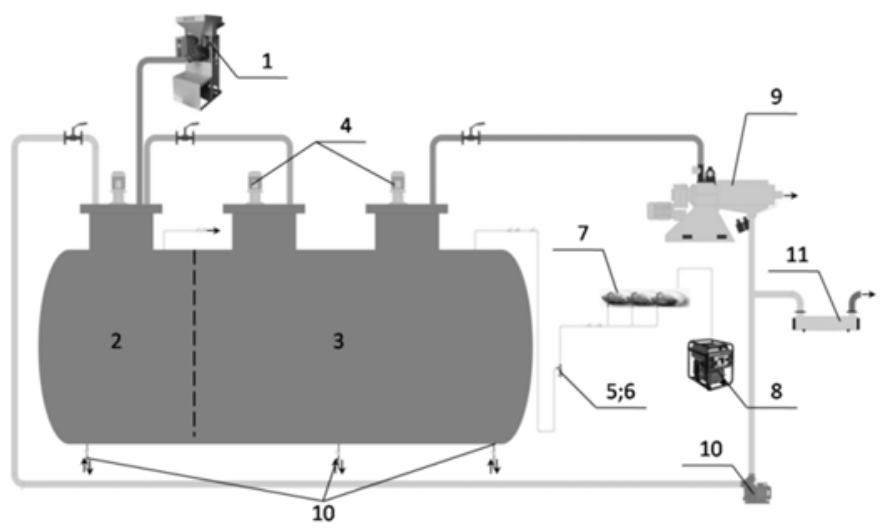

Figure 8: The BGP-100 flow scheme.

\section{Conclusion}

The potential of biomasses of different origin in Russian Federation is significant. The biomass annual growth is comparable with the traditional organic energy consumption, so the modern biotechnology introduction is important.

The performed study of the effect of various factors on the biological treatment process for certain type of biomass (cattle manure) shows that it is possible to reduce processing time and to increase the biomass conversion degree at moderate energy consumption.

It was developed a biogas plant (BGP-100) with a high degree of thermal protection and heat recovery to make the process of biological treatment of organic waste effective even in areas with severe climate conditions.

\section{References}

[1] Baader, V., Done, E., Brennderfer, M. Biogas: Theory and Practice: Translation from German. Kolos, Moscow, 2002, p. 148.

[2] Arbuzova, E.V. \& Shcheklein, S.E. About the problem of energy efficiency of biogas technologies in the climatic conditions of Russia. International scientific journal for alternative energy and ecology, 7, 2011, pp. 108-110.

[3] Klimova, V., Shcelomentsev, I., Burdin, I. \& Shcheklein, S. "Numerical simulation of hydraulic processes in anaerobic bioreactors" WIT Transactions on Ecology and the Environment, Vol. 190, WIT Press, 2014, ISSN 1743-3541.

[4] Shcheklein, S.E., Chugunov, A.V. \& Arbuzova, E.V. “Anaerobic conversion of biomass upon disintegration in ultrasonic fields of low intensity” WIT Transactions on Ecology and the Environment, Vol. 190, WIT Press, 2014, ISSN 1743-3541.

[5] J. Bailey., D. Ollis. Biochemical Engineering Fundamentals. Lane. Translated from English. In 2 parts. Part 2, M: World, 1989, 590 pp. 
[6] V. V. Biryukov. Fundamentals of Industrial Biotechnology. M: KolosS, 2004, 296 pp.

[7] E. S. Pantskhava, V. A. Pogarnov. The contribution of biomass to global energy production. "Commercial Biotechnology" 2003, p. 114.

[8] A. J. Gaab. Prospects for the use of biogas in the example of the Oryol region/Collection of reports of international scientific and technical conference dedicated to the 50th anniversary of LSTU "Energy and energy efficiency technologies.” - Lipetsk, Lipetsk State Technical University, 2006, pp. 29-31.

[9] A. Kovalev. Technology and technical-energy study of biogas production in manure management systems, livestock farms: the dissertation of the doctor of technical sciences: 05.14.08. Moscow, 1998, 244 pp. Ill. RSL OD, 71: 99-5/708-9.

[10] E. Y. Sokolov. District heating and heat networks. M: Publisher MEI, 2001. 471 pp. 